\title{
Impact of Mechanical Homeostasis on the Structure and Function of the Skin
}

\author{
Shun Kimura ${ }^{1,2,3}$, Makoto Take ${ }^{1,3}$ and Takashi Tsuji ${ }^{1 *}$ \\ ${ }^{1}$ Laboratory for Organ Regeneration, RIKEN Center for Developmental Biology (CDB) and RIKEN Center for Biosystems Dynamics Research (BDR), \\ Hyogo, 650-0047, Japan \\ ${ }^{2}$ ROHTO Pharmaceutical Co., Ltd., Osaka, 544-8666, Japan \\ ${ }^{3}$ These authors contributed equally \\ *Corresponding author: Takashi Tsuji, Laboratory for Organ Regeneration, RIKEN Center for Developmental Biology (CDB) and \\ RIKEN Center for Biosystems Dynamics Research (BDR), Japan.
}

To Cite This Article: Shun Kimura, Makoto Takeo, Takashi Tsuji, Impact of Mechanical Homeostasis on the Structure and Function of the Skin. Am J Biomed Sci \& Res. 2021 - 12(2). AJBSR.MS.ID.001721. DOI: 10.34297/AJBSR.2021.12.001721.

Received: 眥 February 22, 2021; Published: 眥 February 26, 2021

\begin{abstract}
Objectives
Tensional homeostasis is crucial for the morphological and functional properties of organs. Skin plays essential roles, such as waterproofing and thermoregulation. The balance of traction force is thought to contribute to the formation of strong and pliable physical structures in organs. In this mini review, we introduce our recent progress showing the importance of the balance of horizontal tension forces to proper skin structure and function by developing a functional human skin equivalent, a tensionally homeostatic skin model.
\end{abstract}

Keywords: Tensional Homeostasis; Skin Equivalent; Traction Force; Mrtf-A; Tensionally Homeostatic Skin Model

Abbreviations: ECM: Extracellular Matrix; MRTF-A: Myocardin-Related Transcription Factor A, IOS: Integumentary Organ System; HSEs: Human Skin Equivalents; THS Model: Tensionally Homeostatic Skin Model; ATRA: All-Trans Retinoic Acid; Itg $\alpha$ 2: Integrin $\alpha 2$

\section{Introduction}

Environmental mechanical stresses, including tension and traction forces, play important roles in the specific morphological and physical properties of tissues and organs [1-3]. Cells sense the physical presence of tension from the surrounding environment, such as the ECM and neighboring cells, via adhesion molecules and the actin cytoskeleton, and they respond to mechanical biological stimuli through mechanotransducers, including MRTF-A [4]. The skin is composed of the epidermis, dermis and skin appendages and plays essential roles, such as waterproofing and thermoregulation. Tensional homeostasis is thought to play important roles in maintaining skin organ homeostasis via mechanical stress signals. Recently, 3D HSEs have been used as an alternative to animal models for safety assessment in skin research and drug discovery $[5,6]$. The challenge was to reproduce more advanced human skin structure and functionality as an alternative to animal experiments [7].

\section{Tensional Homeostasis in the Skin}

The physical stimuli applied to the skin include not only external factors such as compression and tension but also internal factors caused by the traction forces among cells exerted through collagen fibers inside the tissue, termed tensional homeostasis [8]. Because the vector of the tensile force matches the morphological pattern of the skin, such as the orientation of collagen fibers in the dermis and hair follicles, it is considered to play an important role in the structure and function of the skin. In fact, when skin is surgically extracted, contraction and large-scale morphological changes occur, suggesting that tensional homeostasis in the skin is involved in skin homeostasis [9]. However, the role of tensional homeostasis in the 
structure and function of the skin is not fully understood, partially because of the lack of a suitable model to explore this point.

\section{Development of Tensionally Homeostatic Skin Model}

To address the above point, we first aimed to develop an HSE that mimics tensional homeostasis. The conventional Bell-type model, which is widely used for safety assessment worldwide, is created by solidifying a collagen gel mixed with dermal fibroblasts and then seeding epithelial cells on it. However, in this model, the contraction of the dermal part occurs uniformly and results in shrinkage like that in surgically extracted skin, resulting in the loss of tensional homeostasis. Therefore, we developed a culture method that prevents tissue contraction by fixing the outer circumference of collagen gel mixed with dermal fibroblasts to a culture vessel. This tensionally homeostatic skin model (THS model) shows contraction similar to that of natural skin when released from the culture vessel, indicating that tensional homeostasis is reproduced in this skin model.

\section{Tensional Homeostasis Improved the Structure and Function of HSE}

In the THS model, fibroblasts and collagen fibers were oriented according to the direction of tension, similar to natural skin and in contrast to the Bell-type model (Figure 1a). Although the barrier function of the epidermis shows a similar tendency in both the THS model and the Bell-type model, significant promotion of epidermal keratinocyte proliferation was observed in the THS model. Moreover, fibrogenesis of collagen and elastin, which play an important role in the formation of wrinkles and sagging, improved in the THS model. Furthermore, in the THS model, the expression of enzymes involved in the synthesis of collagen and hyaluronic acid and the formation of collagen fiber was significantly upregulated in response to all-trans retinoic acid (ATRA), which effectively counteracted the aging-related decrease in dermal extracellular matrix (Figure 1b). Tensional homoeostasis is known to regulate cell function and fate determination through mechanical stress signals $[6,8]$. We investigated the relationships between mechanical stress signaling and traction force balance in these models. We found that fibroblasts receive tensional stimuli through integrin $\alpha 2$ and MRTF-A, resulting in upregulation of ECM synthesis in the dermis. These results clearly suggest that tensional homeostasis through the Itg $\alpha 2$-MRTF-A axis has an impact on the structure and function of the skin.

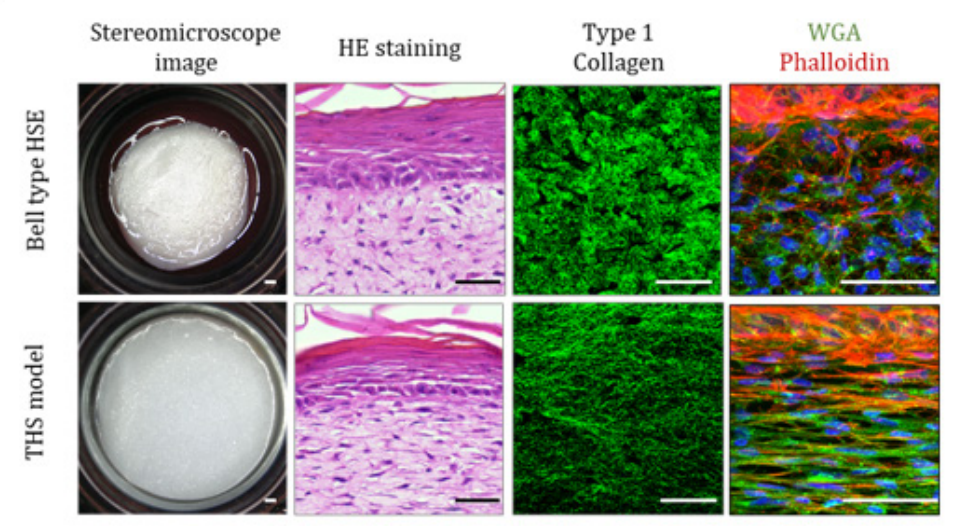

b
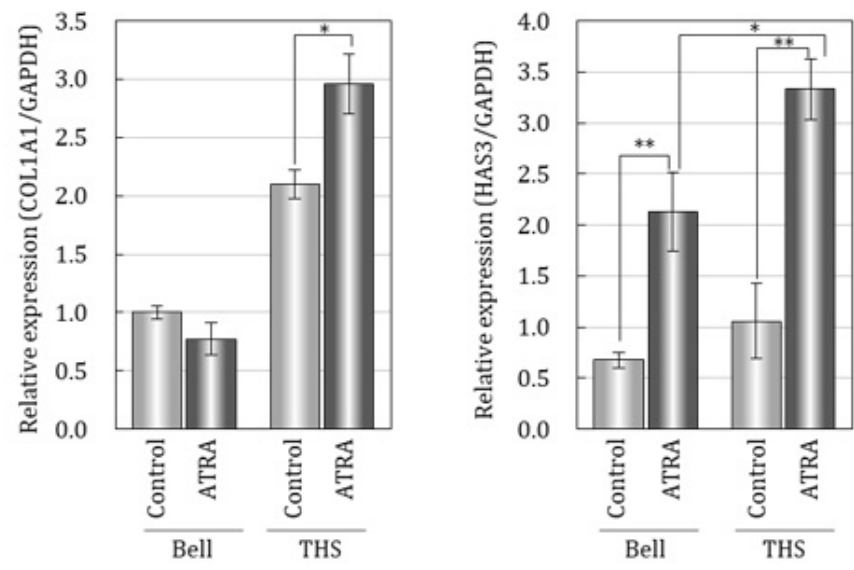

Figure 1: Tensional homeostasis regulates skin morphology and responsibility for ATRA.

a. Macroscopy images and histological analysis by HE Staining and immunohistochemistry for indicated markers. Scale bars, $50 \mathrm{~mm}$.

b. Gene expression analysis for indicated markers without or with ATRA. * $p<0.01$ and ${ }^{* *} p<0.001$, as assessed by Tukey-Kramer test following two-way analysis of variance (ANOVA; $p<0.001$ ); error bars represent the standard deviation modified from Kimura et al., (2021)[9]. 


\section{Conclusion}

This study revealed that tensional homeostasis plays an important role in controlling not only the tissue structure of the skin but also its physiological functions. As an alternative to animal experiments and human clinical trials, our THS model will contribute to the efficient screening and functional analysis of active ingredients, as well as to the development of products with greater support from scientific evidence in the healthcare research field. Furthermore, elucidating the molecular mechanism underlying tensional homeostasis and optimizing the tensional environment of HSEs will open new avenues to cure aging and diseases.

\section{Acknowledgement}

We thank lab members in RIKEN BDR. This work was supported by JSPS KAKENHI (Grant number: JP16H01851 to T. T.). We would like to thank Rohto Pharmaceutical Co., Ltd., and Organ Technologies Inc. for their funding support.

\section{Conflict of Interest}

T.T. is a supreme technical advisor at Organ Technologies Inc. This work was partially performed under the condition of an Invention Agreement between RIKEN, Rohto Pharmaceutical Co., Ltd., and Organ Technologies Inc.. The remaining authors declare no competing interests.

\section{References}

1 DuFort CC, Paszek MJ, Weaver VM (2011) Balancing forces: architectural control of mechanotransduction. Nat Rev Mol Cell Biol 12(5): 308-319.

2 Mammoto T, Ingber DE (2010) Mechanical control of tissue and organ development. Development 137(9): 1407-1420.

3 Sean Porazinski, Huijia Wang, Yoichi Asaoka, Martin Behrndt, Tatsuo Miyamoto, et al. (2015) YAP is essential for tissue tension to ensure vertebrate 3D body shape. Nature 521(7551): 217-221.

4 Mammoto A, Mammoto T, Ingber DE (2012) Mechanosensitive mechanisms in transcriptional regulation. J Cell Sci 125(13): 3061-3073.

5 Zhang Z, Michniak Kohn BB (2012) Tissue engineered human skin equivalents. Pharmaceutics 6(4): 26-41.

6 Mathes SH, Ruffner H, Graf Hausner U (2014) The use of skin models in drug development. Adv Drug Deliv Rev 69-70.

7 N Ali M Hosseini, S Vainio, A Taïeb, M Cario André HR Rezvani (2015) Skin equivalents: skin from reconstructions as models to study skin development and diseases. Br J Dermatol 173(2): 391-403.

8 Chao Kai Hsu, His Hui Lin, Hans I Chen Harn, Michael W Hughes, Ming Jer Tang, et al. (2018) Mechanical forces in skin disorders. J Dermatol Sci $90(3): 232-240$

9 Shun Kimura, Ayako Tsuchiya, Miho Ogawa, Miki Ono, Nao Suda, et al. (2020) Tissue-scale tensional homeostasis in skin regulates structure and physiological function. Commun Biol 3(1): 637. 